\title{
Premature lethality, hyperactivity, and aberrant phosphorylation in transgenic mice expressing a constitutively active form of Fyn
}

\author{
Di Xia and Jürgen Götz* \\ Clem Jones Centre for Ageing Dementia Research, Queensland Brain Institute, The University of Queensland, Brisbane, QLD, Australia
}

Edited by:

Peter Giese, King's College London, UK

\section{Reviewed by:}

Gerald Muench, Cook University, Australia

Gloria Lee, University of lowa, USA

*Correspondence:

Jürgen Götz, Clem Jones Centre for Ageing Dementia Research,

Queensland Brain Institute, The

University of Queensland, St Lucia

Campus, Brisbane, OLD 4072,

Australia

e-mail:j.goetz@uq.edu.au
The kinase Fyn, the microtubule-associated protein tau and the peptide amyloid- $\beta$ (A $\beta$ ) constitute a toxic triad in Alzheimer's disease (AD). Tau's subcellular localization is mainly regulated by phosphorylation whereas Fyn's localization is dictated by palmitoylation targeting it to the plasma membrane in a reversible manner. We have previously shown that tau is required for Fyn to be targeted to the dendritic spine. We had also shown that a truncated form of tau ( $\Delta$ tau) that accumulates in the cell soma is capable of trapping Fyn and preventing it from entering the spine. Here we determined that palmitoylation is required for Fyn's membrane and spine localization. We further evaluated the functional consequences of neuronal over-expression of the constitutively active Y531F mutant form of Fyn (FynCA) in transgenic mice. We found that the FynCA transgenic mice displayed a reduced weight, a massively reduced lifespan and a high level of hyperactivity. The lifespan of the FynCA mice was only slightly extended by crossing them with $\Delta$ tau transgenic mice, possibly reflecting differences in expression patterns of the transgenes and high levels of transgenic FynCA compared to endogenous Fyn. Analysis of synaptosomes revealed that FynCA accumulated at high levels in the spine, resulting in increased levels of the NMDA receptor subunit NR2b phosphorylated at residue Y1472. Tau was strongly phosphorylated at the AT8 epitope S202/T205 as shown by Western blot and immunohistochemistry indicating that an increased tyrosine kinase activity of Fyn has down-stream consequences for serine/threonine-directed phosphorylation.

Keywords: Alzheimer, dendrite, Fyn kinase, palmitoylation, phosphorylation, spine, tau

\section{INTRODUCTION}

In Alzheimer's disease ( $\mathrm{AD})$, serine/threonine-directed phosphorylation has attracted significantly more attention than tyrosinedirected phosphorylation (Götz et al., 2013). One of the reasons is that the microtubule-associated protein tau, a protein implicated in $\mathrm{AD}$, contains 80 serines and threonines, many of which have been shown to be phosphorylated under disease conditions, compared to only 5 tyrosine residues (Chen et al., 2004). When tau transgenic mice as a model of $\mathrm{AD}$ are analyzed this routinely includes the assessment of the phosphorylation status of relevant serine and threonine residues, but rarely that of tyrosines. Similarly, to establish the sequence of tau-related cytoskeletal changes in $\mathrm{AD}$, the serine/threoninedirected antibody AT8 and not a tyrosine-directed antibody has been used (Braak et al., 1994). However, it is increasingly appreciated that tyrosine phosphorylation has also an important role in $\mathrm{AD}$ (Boehm, 2013). Of tau's five tyrosine residues, Y18 is particularly interesting because it is specifically phosphorylated by the tyrosine-directed kinase Fyn, a member of the Src family of non-receptor tyrosine kinases (http://cnr.iop.kcl.ac.uk/hangerlab/tautable) (Figure 1A).

Under physiological conditions, the majority of tau is localized to the axon, whereas tau's "counterpart," MAP2, is mainly localized to the dendrite. However, as we have found recently, tau is also localized, albeit at lower levels, to the dendrite where it serves an important function that cannot be taken over by MAP2 (Ittner et al., 2010): Tau is required to target Fyn to the dendritic spine where it phosphorylates the NMDA receptor, a prerequisite for recruiting the postsynaptic density (PSD) $(95 \mathrm{kD})$ protein $(\mathrm{PSD}-95)$ into a protein complex. This complex then mediates the excitotoxic signaling triggered by $A \beta$, a peptide that aggregates and forms amyloid plaques in $\mathrm{AD}$ brains. In the absence of tau or when a form of tau is expressed that accumulates in the cell soma and is excluded from the dendrite $(\Delta$ tau, Figure 1B), Fyn is prevented from entering the spine and thereby, $A \beta$ cannot excitotoxically signal through the NMDA receptor (either directly, or indirectly) any more (Ittner et al., 2010). When a full-length form of tau is expressed that carries the pathogenic P301L mutation found in frontotemporal dementia, excitotoxic signaling is augmented because of an increased dendritic localization of phosphorylated mutant tau and consequently, increased levels of dendritic Fyn (Ittner and Götz, 2011). These data are supported by the finding that $A \beta$ causes missorting of endogenous tau into the dendritic compartment (Zempel et al., 2010). Not surprisingly, overexpression of wild-type murine Fyn induces synaptic and cognitive impairments in a transgenic mouse model with $A \beta$ accumulation (Chin et al., 2005), and deletion of Fyn ameliorates some 

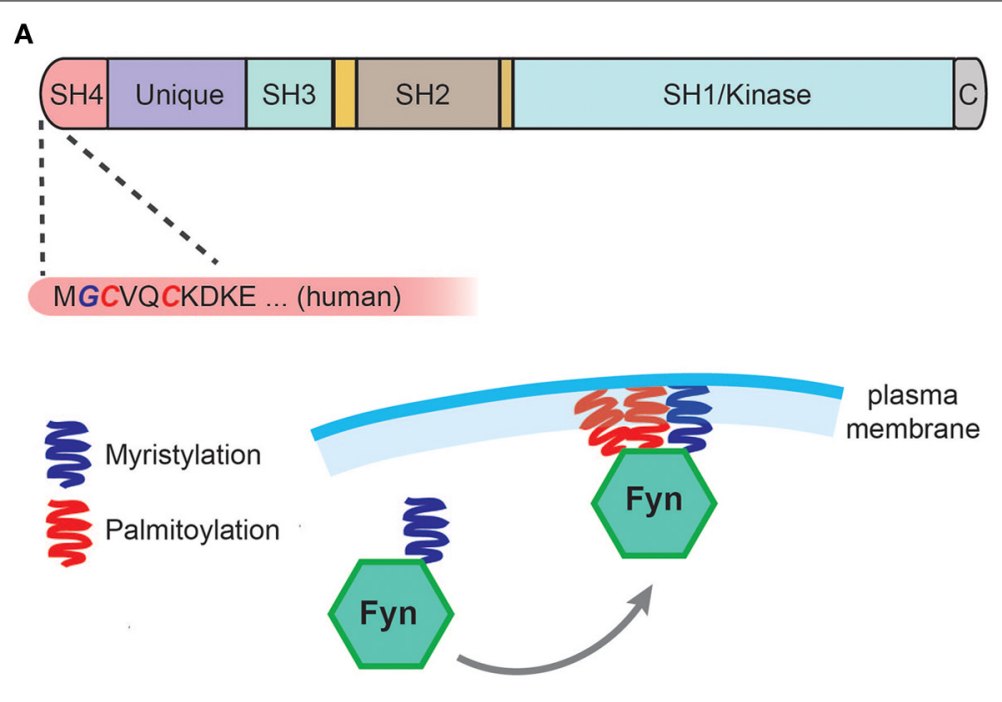

B
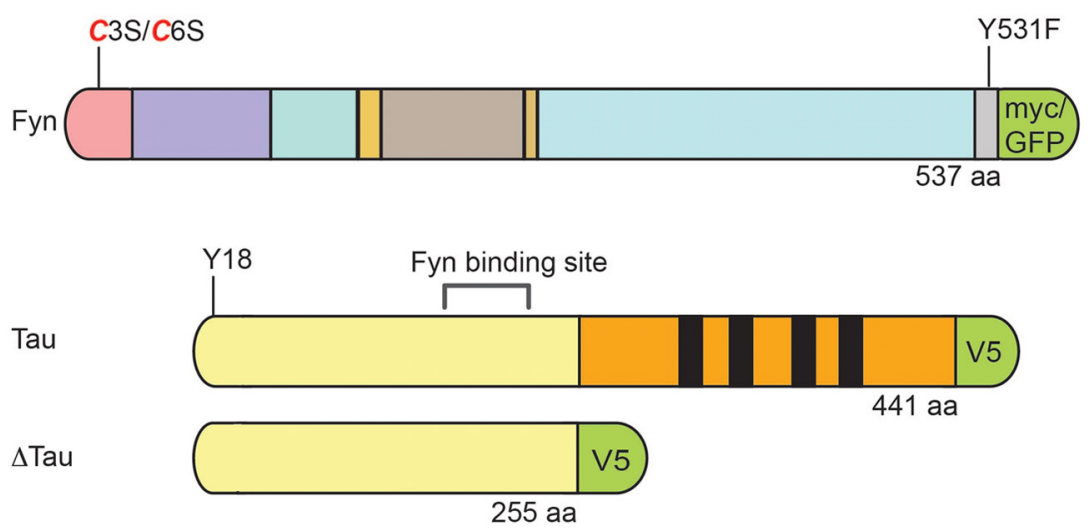

FIGURE 1 | Structure of Fyn, its membrane localization, and constructs used for expression of Fyn, tau and $\mathbf{\Delta}$ Tau. (A) Fyn is a $59 \mathrm{kDa}$ protein that contains an amino-terminal Src-homology $(\mathrm{SH})$ region with acylation sites, a unique domain, an $\mathrm{SH} 3$ domain (with which it interacts with PXXP motifs), an $\mathrm{SH} 2$ domain (with which it interacts with phospho-tyrosine), an $\mathrm{SH} 1 /$ kinase domain, and a carboxy-terminal regulatory tail. The most amino-terminal glycine residue can be myristoylated, which occurs co-translationally on free ribosomes. In addition, Fyn's amino-terminal cysteines C3 and C6 can be palmitoylated, and it is this acylation reaction that anchors the kinase to the plasma membrane. (B) Expression constructs for human Fyn were either wild-type, C3S/C6S to prevent palmitoylation, or Y531F to generate a constitutively active form of Fyn (FynCA). Tau was expressed either as full-length [with the longest human tau isoform, hTau40, containing four microtubule-binding domains (MBDs) indicated in black] or a truncated form ( $\Delta$ tau) lacking the MBDs. For detection of tau and Fyn, myc, GFP and V5 were used as tags as indicated. of the phenotypes induced by $A \beta$ (Chin et al., 2004). Together, this established the notion of a toxic triad of the three molecules, tau, Fyn and $A \beta$ (Haass and Mandelkow, 2010), presenting $A \beta$ as the trigger, and tau as the bullet in $\mathrm{AD}$ pathogenesis (Bloom, 2014).

Fyn is a $59 \mathrm{kDa}$ protein that exists as two isoforms due to alternative splicing of exon 7, with FynT being expressed in T-cells, and FynB in the brain and additional organs (Kramer-Albers and White, 2011). Fyn contains, in this order, an amino-terminal Srchomology $(\mathrm{SH})$ region with acylation sites, a unique domain, an SH3 domain (with which it interacts with PXXP motifs), an $\mathrm{SH} 2$ domain (with which it interacts with phospho-tyrosine), an SH1/kinase domain, and a carboxy-terminal regulatory tail (Figure 1A). The most amino-terminal glycine residue can be myristoylated, which occurs co-translationally on free ribosomes.
In addition, Fyn's amino-terminal cysteines, C3 and C6, can be palmitoylated, and it is this acylation reaction that anchors the kinase to the plasma membrane. Palmitoylation in general enhances the hydrophobicity of proteins and contributes to their membrane association. In contrast to myristoylation, palmitoylation is usually reversible (because the bond between palmitic acid and protein is often a thioester bond) (Figure 1A). A good example of another palmitoylated protein in the dendritic spine is PSD-95. Interestingly, palmitoylation of Fyn seems to direct the SH4 domain away from the membrane, which may be important for the interaction of Fyn with target proteins (Rawat and Nagaraj, 2010).

The activity of Fyn is regulated by differential phosphorylation at multiple sites (Kramer-Albers and White, 2011). In its inactive state, the regulatory tyrosine at the carboxy-terminus 
(Y531 in mouse FynB) is phosphorylated, forming an intramolecular bond with the $\mathrm{SH} 2$ domain of the kinase. This phosphorylation negatively regulates kinase activity (Nada et al., 1993). Furthermore, the SH3 domain binds intra-molecularly to a linker region located between the $\mathrm{SH} 2$ and the kinase domain. This keeps the kinase in an inactive closed conformation in which both protein binding domains are occupied. Upon dephosphorylation of the carboxy-terminal regulatory tyrosine residue by tyrosine phosphatases, the intramolecular binding to the $\mathrm{SH} 2$ domain is abolished. Loss of SH2-dependent folding alters the conformation of the kinase to generate a more open form making the $\mathrm{SH} 2$ and $\mathrm{SH} 3$ domains available for downstream protein interactions. Furthermore, the open conformation enables an intermolecular auto-phosphorylation of Y420 that stabilizes the active state of the catalytic site (Roskoski, 2004). Src kinases can also be stimulated by interfering with the inhibitory intramolecular binding of $\mathrm{SH} 2$ and $\mathrm{SH} 3$ domains which both can bind to external ligands leading to an activation of the kinase (Ostareck-Lederer et al., 2002; Sette et al., 2002).

Tau interacts with Fyn at least in two ways. Firstly, Y18 is phosphorylated by Fyn, and the phosphorylated motif interacts with Fyn via Fyn's SH2 domain (Usardi et al., 2011). Secondly, Tau contains seven PXXP motifs located in the amino-terminus (all of which are retained in the $\Delta$ tau construct). Of these, the seventh proline-rich RTPPKSP motif has been shown to be crucial for the interaction with the SH3 domain of Fyn and other Src non-receptor tyrosine kinases (Lee et al., 1998; Bhaskar et al., 2010; Ittner et al., 2010). Interestingly, this motif is also criti$\mathrm{cal}$ in the interaction of tau with the heterotrimeric phosphatase PP2A (Sontag et al., 2012). The PP2A regulatory subunit $\mathrm{B} \alpha$ binds to and dephosphorylates tau, and thereby regulates microtubule stability (Kins et al., 2001; Sontag et al., 2012). When Fyn is bound to the (seventh) proline-rich RTPPKSP motif that is conserved in both tau and MAP2, this inhibits the interaction of $\mathrm{PP} 2 \mathrm{~A} / \mathrm{B} \alpha$ with either tau or MAP2. The corresponding synthetic RTPPKSP peptide, but not the phosphorylated RpTPPKSP version, competes with tau and MAP2 for binding to PP2A/B $\alpha$. This finding is remarkable because the down-regulation of $\mathrm{PP} 2 \mathrm{~A} / \mathrm{B} \alpha$ and the deregulation of Fyn/tau interactions have been linked to enhanced tau phosphorylation in AD (Sontag et al., 2012). Why in tau knock-out mice, MAP2 that is mainly a dendritic protein cannot compensate for the absence of tau and target Fyn to the dendritic spine is not understood, especially as both MAP2 and tau can efficiently bind Fyn (Sontag et al., 2012; Lashuel et al., 2013). Not only tau but also MAP2 have been shown to bind to Fyn via Fyn's SH3 domain (Zamora-Leon et al., 2001).

Here, we aimed to address how Fyn is localized subcellularly in vitro and what the functional consequences are of the transgenic overexpression of a constitutively active form of Fyn, Y531F.

\section{MATERIALS AND METHODS MUTAGENESIS}

The C3S/C6S double mutation was introduced by PCR into the lentiviral vector Fyn-myc pLUV6 that contains the human Fyn isoform 1 cDNA fused to a myc tag (Ittner et al., 2010). The resultant mutant construct was subcloned into the pGEM-T easy vector by TA ligation (Promega), followed by ApaI/SalI digestion and ligation into the pEGFP-N1 vector (Clontech) to generate a C3S/C6S Fyn-EGFP construct. Similarly, the Y531F mutation was cloned by PCR, with or without a myc-tag, and subcloned via a unique XhoI site into the murine Thy1.2 expression vector $\mathrm{pEX} 12$ for subsequent neuronal expression in transgenic mice (Ittner and Götz, 2007). The plasmids were sequenced using the service of the AEGRC sequencing facility (University of Queensland). The resulting transgenic mice were labeled FynCA.

\section{CELL CULTURE}

HEK293T cells were cultured in DMEM medium, supplemented with $2 \mathrm{mM}$ Glutamax (Life Technologies) and 10\% FBS (Sigma). Cells were seeded as $50 \%$ confluency in $6 \mathrm{~cm}$ culture dishes and allowed to grow for $24 \mathrm{~h}$ before transfection. For high efficiency transfection, lipofectamine LTX (Invitrogen) was used in a 2:1 ratio to DNA. Hippocampal neurons from E18 wildtype or Tau knock-out (Tucker et al., 2001) mouse pups were plated onto poly-D-lysine coated coverslips in a 12-well plate at a density of 5000 cells/well. As a plating medium, Neurobasal medium was used, supplemented with 5\% FBS (Hyclone), 2\% B27 (Life Technologies), $2 \mathrm{mM}$ Glutamax, and $50 \mathrm{U} / \mathrm{mL}$ penicillin/streptomycin. Neurons were switched to serum-free Neurobasal medium $24 \mathrm{~h}$ post-seeding and half the medium was changed twice a week. Neurons were transfected at DIV 18 using lipofectamine 2000 (Invitrogen).

\section{GENERATION OF TRANSGENIC MICE}

FynCA transgenic mice were generated by pronuclear microinjection as described previously (Ittner and Götz, 2007). $\Delta$ tau74 mice have been generated previously, by removing amino acids 256-441 from the longest human tau isoform, htau40, and expressing this truncated form of tau under control of the murine Thy1.2 promoter (Ittner et al., 2010). As a tau knockout strain, mice were used that have a GFP cassette inserted in frame into the first coding exon, resulting in a fusion protein that contains the first 31 amino acids of tau (Tucker et al., 2001). Animal experimentation has been approved by the Animal Experimentation Committee of the University of Queensland (QBI/327/11/NHMRC/ARC/BREED, QBI/027/12/ NHMRC).

\section{IMMUNOHISTOCHEMISTRY}

Mice were immersion fixed in 4\% PFA rather than perfused because of their small size, brains embedded in paraffin and $7 \mu \mathrm{m}$ sections obtained as described (Deters et al., 2008). For antigen retrieval, the sections were microwaved in AR buffer (Dako Envision Flex Target Retrieval Solution low pH \#K8005) for $15 \mathrm{~min}$ on low power before the buffer started to boil. The sections were then left to cool for $1 \mathrm{~h}$ in the AR buffer at RT. Blocking was done in $20 \%$ FBS, $1 \%$ BSA in TBST for $1 \mathrm{~h}$ at RT. Primary antibodies were used over night at $4^{\circ} \mathrm{C}$ and secondary antibodies for $1.5 \mathrm{~h}$ at RT. Primary antibodies were Myc-Tag rabbit mAb (Cell Signaling Technologies, \#71D10, used at 1:100) and anti-Human PHF-Tau Monoclonal Antibody AT8 (Thermo Fisher, MN1020, used at 1:400). Secondary antibodies were polyclonal goat anti-rabbit IgG biotinylated (Dako, \#E0432, used at 1:500) and polyclonal rabbit anti-mouse IgG biotinylated (Dako, 
\#E0413, used at 1:500). The VectaStain Elite ABC Kit \#PK6102 was used and Envision Flex DAB chromogen (Dako, \#DM827) and Envision Flex Substrate Buffer (Dako, \# DM823) for DAB development. Haematoxylin was used for counter-staining.

\section{SYNAPTOSOMAL EXTRACTION AND WESTERN BLOT ANALYSIS}

Synaptosomes from the mouse forebrain $(n=4$ for the three genotypes) were prepared using a modified volume-adjusted protocol based on a protocol described previously (Ittner et al., 2010). In brief, the tissue was homogenized in sucrose buffer $\left(1 \mathrm{ml} / 60 \mathrm{mg}\right.$ tissue. $0.32 \mathrm{M}$ sucrose, $1 \mathrm{mM} \mathrm{NaHCO}_{3}, 1 \mathrm{mM}$ $\mathrm{MgCl}_{2}, 0.5 \mathrm{mM} \mathrm{CaCl}$ ) using a tissue homogenizer. Brain homogenate was centrifuged at $1400 \mathrm{~g}$ for $10 \mathrm{~min}$ and cleared by centrifugation at $720 \mathrm{~g}$ for $10 \mathrm{~min}$. $200 \mu \mathrm{L}$ of supernatant was collected as whole brain lysate and the pellet containing cell debris and nuclei was discarded. The remaining supernatants were then centrifuged at $13,800 \mathrm{~g}$ for $10 \mathrm{~min}$ to obtain the crude synaptosomes in the pellet $(\mathrm{P})$. The pellet was resuspended in $200 \mu$ l Sucrose Buffer, layered over $1.8 \mathrm{~mL}$ pre-cooled $5 \%$ Ficoll and centrifuged at 45,000 g for $45 \mathrm{~min}$. The supernatant was then removed carefully and the pellet resuspended in $200 \mu \mathrm{L}$ pre-cooled 5\% Ficoll, which was then layered over $1.8 \mathrm{~mL}$ pre-cooled 13\% Ficoll and centrifuged at 45,000 g for $45 \mathrm{~min}$. A milky interface containing the synaptosomes was recovered, topped-up with 5\% Ficoll to $2 \mathrm{~mL}$ and centrifuged at $45,000 \mathrm{~g}$ for $25 \mathrm{~min}$ to pellet the synaptosomes. Purified synaptosomes were further extracted sequentially with buffers of increasing stringency: pH6 (40 mM Tris- $\mathrm{HCl}, 2 \%$ Triton X-100, $0.5 \mathrm{mM}$ $\mathrm{CaCl}_{2}$ ); pH8 (20 mM Tris-HCl, $1 \%$ Triton X-100); and SDS (5\% SDS). The SDS buffer extract contains strongly PSD-associated proteins. This fraction was named "PSD." All centrifugations were carried out at $4^{\circ} \mathrm{C}$. For Western blot analysis, the following primary antibodies were used: total Tau (Dako, polyclonal, 1:5,000), Fyn (Fyn15; monoclonal, Santa Cruz, 1:500), PSD-95 (Millipore, 1:2,000), GAPDH (polyclonal, Millipore, 1:5,000), NR2b (polyclonal, Millipore, 1:1,000), pTyr ${ }^{1472}$ - NR2b (polyclonal, Sigma, 1:1,000), Myc-tag (71D10, rabbit monoclonal, 1:2,000), AT8 (phospho-tau, pSer202 + pThr205, monoclonal, Thermo, 1:1,000), 12E8 (phospho-tau pSer262 (+ pS356)), polyclonal, Thermo, 1:4,000), HT7 (human Tau, monoclonal, Thermo, 1:2,000), and Actin (monoclonal, Millipore, clone C4, monoclonal, 1:5,000).

\section{RESULTS}

\section{PALMITOYLATION OF Fyn IS REOUIRED FOR SPINE LOCALIZATION}

Fyn contains two cysteines (C3 and C6) that are acetylated in a palmitoylation reaction. To determine the role of these residues in subcellular localization we mutated the palmitoylation signal by replacing the two cysteines by serines as described (Wolven et al., 1997), and equipped Fyn with a GFP tag. When transfected into HEK293 cells, this revealed a membrane association for wildtype Fyn (Figure 2A). Fyn(C3S/C6S), in contrast, was excluded from the plasma membrane and accumulated intracellularly in endosomes (Sandilands et al., 2007) (Figure 2B). We next determined the subcellular localization in primary hippocampal cultures obtained from wild-type mouse embryos. After 21 days (DIV2) in culture, the neurons displayed a myriad of spines.
We co-transfected the cultures with RFP and either Fyn-GFP or Fyn(C3S/C6S)-GFP and found that wild-type Fyn was localized to spines (Figures 2C-E) whereas Fyn(C3S/C6S)-GFP was excluded from entering the spines (Figures $\mathbf{2 F}-\mathbf{H}$ ). Together this reveals that palmitoylation and hence, membrane association, is required for the localization of Fyn into dendritic spines.

We have previously shown that $\Delta$ tau in $\Delta$ tau74 transgenic mice is localized to the cell body, but excluded from the dendritic compartment (Ittner et al., 2010). This finding was also supported biochemically, by analyzing synaptosomal preparations and comparing $\Delta$ tau localization with that of full-length tau. To determine whether $\Delta$ tau is also excluded from the dendritic compartment and in particular, spines, in primary neuronal cultures, we transfected both full-length tau equipped with a V5 tag (Tau-V5) together with RFP, and $\Delta$ tau-V5 together with RFP, respectively, using primary neuronal cultures derived from tau knock-out mice. This showed that full-length tau is distributed throughout the neuron, but excluded from the spines (Figures 3A-C). In contrast, $\Delta$ tau was dramatically reduced from the dendritic compartment and levels were found to be below detection levels past the secondary branch-point (as indicated by the arrows) (Figures 3D-F). We next determined whether expression of $\Delta$ tau would affect Fyn localization, by co-transfecting wild-type neurons with Fyn-EGFP, RFP, and $\Delta$ tau-V5 (Figures 3G-K). We found that different from $\Delta$ tau74 mice in which transgenic overexpression of $\Delta$ tau (or knocking-out endogenous tau) prevented endogenous Fyn from entering the spines (Ittner et al., 2010), transfecting $\Delta$ tau into primary neurons was not capable of preventing transfected Fyn (Fyn-EGFP) from entering the spine, possibly reflecting differences in expression levels.

\section{MICE WITH A CONSTITUTIVE EXPRESSION OF FYn ARE CHARACTERIZED BY REDUCED WEIGHT, HYPERACTIVITY, AND PREMATURE DEATH}

In brain, Fyn is expressed in both neurons and glial cells, and in both cell-types, tau has an important function in regulating Fyn (Klein et al., 2002; Ittner et al., 2010). To establish a mouse model with constitutive expression of Fyn in neurons, we introduced the Y531F mutation into the human Fyn cDNA and cloned this "FynCA" cDNA into the mThy1.2 expression vector for neuronal expression, generating constructs that either contained or did not contain the myc tag for subsequent detection (Figure 1A). We generated a series of transgenic founders by pronuclear injection of C57BL/ $6 \times \mathrm{DBA} / 2$ oocytes: four $(\# 11,15,16$, and 17) without a myc tag, and $10(\# 4,12,13,14,20,22,24,35,55$, and 58) with a tag. We screened the offspring obtained from these FynCA founder animals by Western blotting and found that whenever the transgene-positive offspring died early (what happened around 3 weeks of age), the transgene was expressed at detectable levels, whereas when the mice did not die at an early age the offspring of that particular founder did not express the transgene at detectable levels (Figure 4A). We specifically identified founders \#14, 35, and 55 as generating offspring characterized by early lethality. The transgene-positive offspring derived from these lines was significantly smaller, as shown for \#55 offspring (Figure 4B), and showed less weight gain than the non-transgenic littermates such that by 2-3 weeks of age they would only have half the weight of 

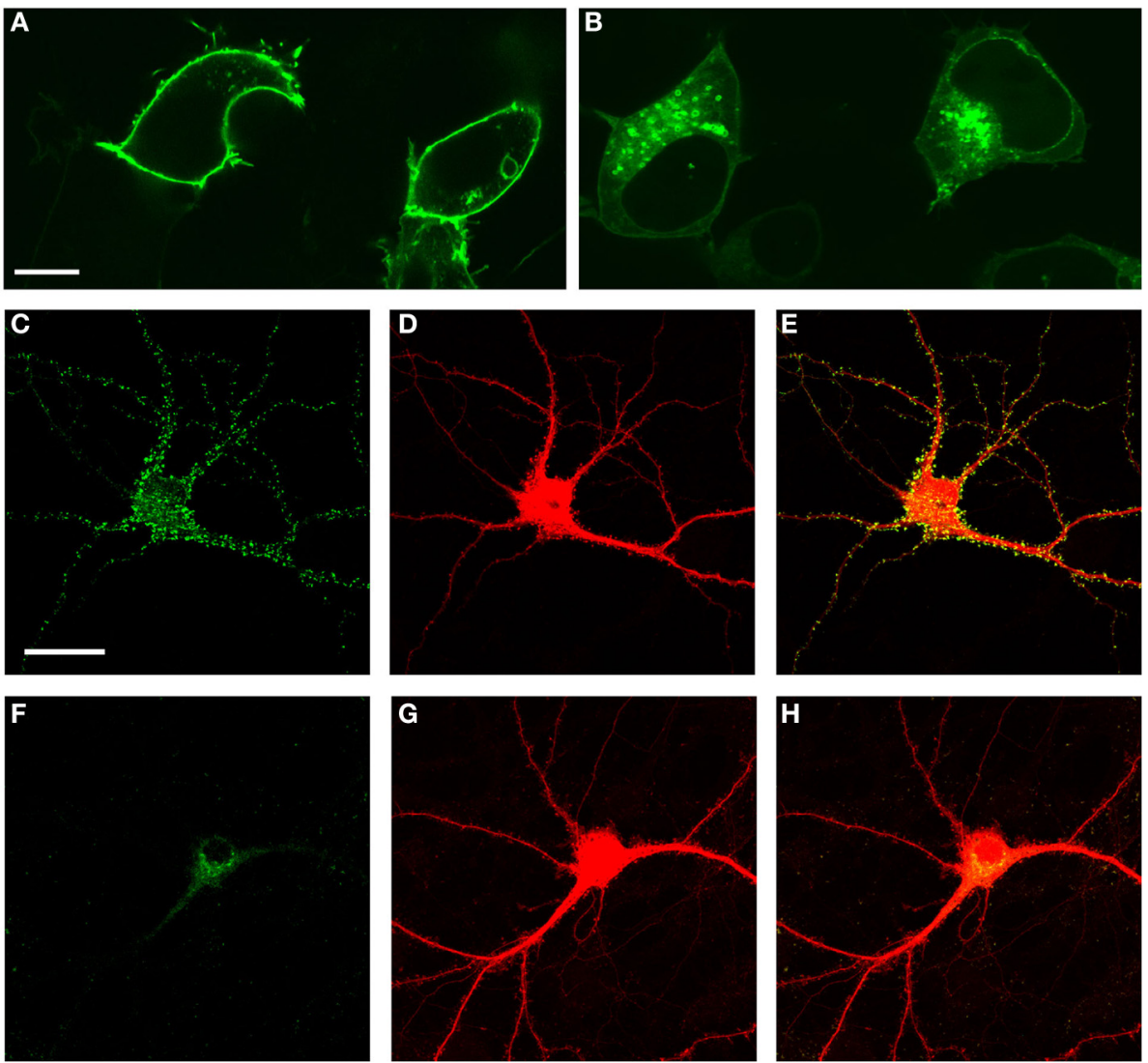

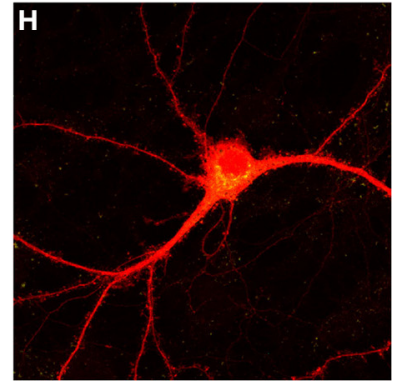

FIGURE 2 | Palmitoylation and hence, membrane association, is required for the localization of Fyn into dendritic spines. Transfection of Fyn expression constructs in HEK293 cells (A,B) and primary hippocampal cultures (C-H) reveals that wild-type Fyn associates with the plasma membrane in HEK293 cells (A), whereas mutating Fyn's two cysteines (C3/C6) that are acetylated in a palmitoylation reaction to cysteines causes Fyn's accumulation intracellularly in endosomes (B). In the neuronal cultures, wild-type Fyn is localized to spines (C-E) whereas Fyn(C3S/C6S)-GFP is excluded from entering the spines (F-H). RFP (D,G) was used to visualize all neuronal structures including the spines. Scale bar: $10 \mu \mathrm{m}$ (A,B); $20 \mu \mathrm{m}$ (C-H). non-transgenic littermates (Figure 4C). The transgenic mice displayed persistent tremor and lack of coordination while moving, as well as pronounced hyperactivity and an altered locomotion behavior (Supplementary movie, shown at 18 days of age). More specifically, when separated from the litter they would start running in circles in the cage for hours, and this could only be halted when the transgenic mice were put back to the litter. Interestingly, only a slight separation from the litter was sufficient to re-instigate this type of behavior, suggesting high levels of anxiety in FynCA mice. To complement the movie, we recorded the path of four mice each aged 18 days of age for $1 \mathrm{~min}$, after the mice had been kept isolated in a box for $1 \mathrm{~h}$ (Figure 4D).

\section{PARTIAL RESCUE OF FynCA MICE BY CROSSING ONTO A $\Delta$ tau BACKGROUND}

We next analyzed the offspring of the FynCA founder animals both by immuno-histochemistry (Figure 5) and Western blotting (Figure 6) using a monoclonal anti-Fyn antibody. The latter revealed a strong expression of Fyn $(59 \mathrm{kDa})$ in mouse brains derived from the FynCA founders \#14, 35, and 55. As shown for offspring of founder \#55, levels of Fyn in FynCA transgenic mice were approximately six-fold higher than those for endogenous murine Fyn in wild-type mice (Figure 6A). The affinity of the antibody for human and murine Fyn is expected not to differ because of a 100\% homology of the epitope used for immunization as well as flanking sequences. We next determined the expression pattern of FynCA in \#55 offspring by immunohistochemistry using a rabbit anti-myc antibody to visualize FynCA expression. We found that FynCA was widely expressed in FynCA transgenic brain (Figure 5A), using non-transgenic littermates as controls (Figure 5B). Prominent expression of the FynCA transgene was found in neurites and fiber tracts as shown for the hippocampus and the fimbria fornix, but the FynCA transgenic protein was also found accumulating in the cell bodies e.g., of large motor neurons in the brain stem and in nuclei such as the pontine nucleus. As tau is a substrate of Fyn we aimed to assess its phosphorylation in the FynCA mice. The phospho-Y18-tau-specific antibody 9G3 could not be employed because it is human tau-specific and does not detect murine tau phosphorylated at Y18 (Bhaskar et al., 2010). We therefore aimed to determine whether Ser/Thr-directed phosphorylation is increased in the presence of constitutively active Fyn. In human tau transgenic mice phosphorylation of Ser/Thr-epitopes of tau only occurs as the mice become older. We nonetheless 

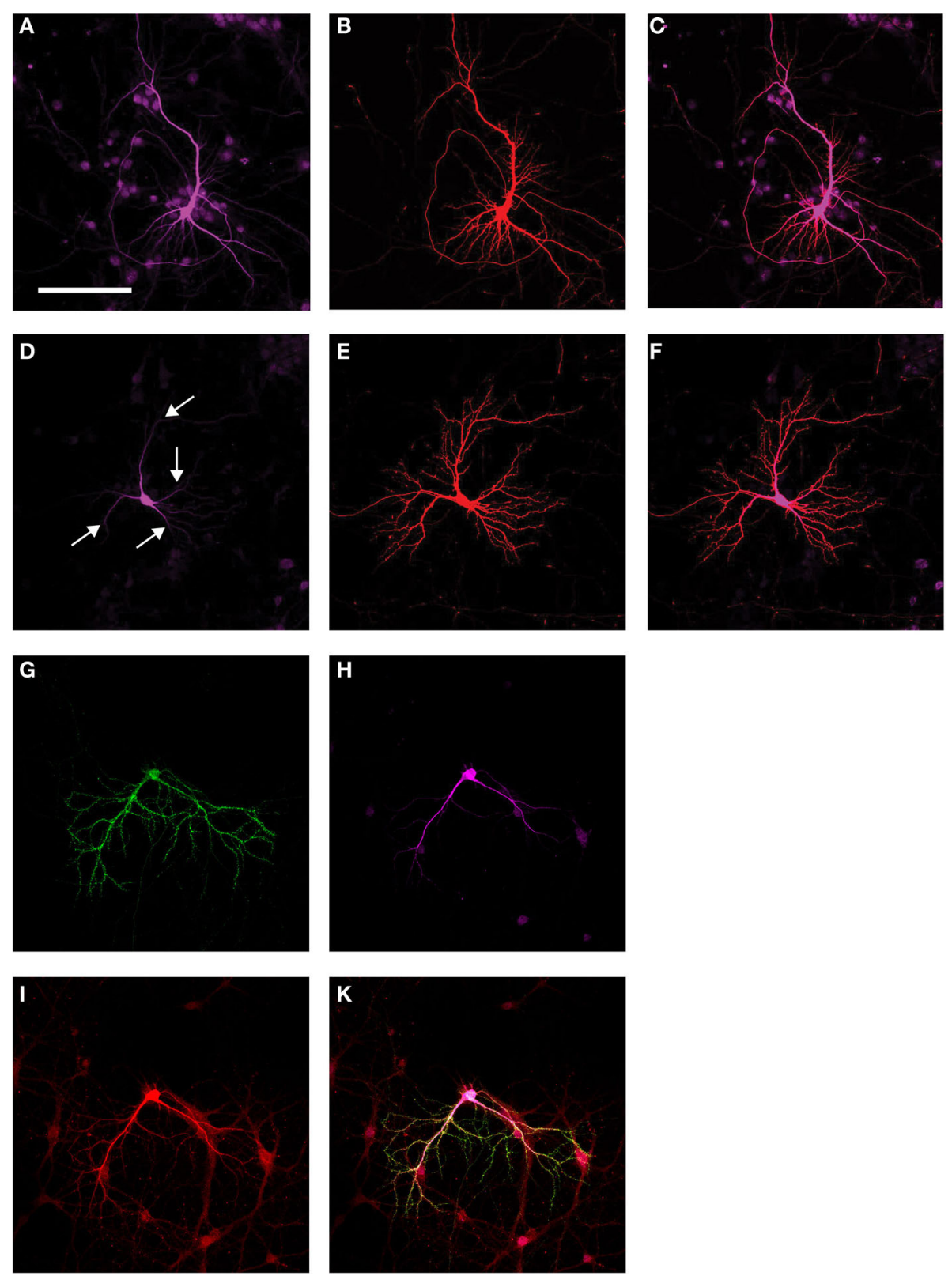

FIGURE 3 | Subcellular localization of Fyn and $\Delta$ tau. Transfection of tau knock-out primary neuronal cultures with a full-length tau construct equipped with a V5 tag (Tau-V5) (A) together with RFP (B) shows distribution of full-length tau throughout the neuron, but spines are excluded. (Merged image: C). Transfection of $\Delta$ tau-V5 (D) together with RFP (E) reveals that $\Delta$ tau is dramatically reduced from the dendritic compartment, and that levels are below detection past the secondary branch-point (as indicated by the arrows) (Merged image: F). When Fyn-EGFP (G) was over-expressed together with $\triangle$ tau-V5 $(\mathbf{H})$ and RFP (I) in wild-type primary neurons, different from $\Delta$ tau 74 mice in which transgenic over-expression of $\Delta$ tau prevents endogenous Fyn from entering the spines, transfecting $\Delta$ tau into primary neurons does not prevent transfected Fyn from entering the spine, possibly reflecting differences in expression levels (Merged image: $\mathbf{K}$ ). Scale bar: $100 \mu \mathrm{m}$ (A-K) analyzed 2 week-old FynCA mice using the phosphotau-specific antibody AT8 (Figure 5C), using non-transgenic littermates as controls (Figure 5D). We found that the mice were strongly phosphorylated at the AT8 epitope in fiber tracts as shown for the hippocampus, and pronounced AT8-immunoreactivity was also seen in cell bodies in several brain areas.

To determine whether expression of $\Delta$ tau would ameliorate the FynCA pathology at least to a degree that would allow us to establish FynCA lines from the founder animals, we bred the FynCA founders with $\Delta$ tau 74 mice. $\Delta$ tau is widely expressed in the brains of $\Delta$ tau74-transgenic mice as revealed with the human tau-specific antibody HT7, whereas no expression is found in non-transgenic littermate controls (Ittner et al., 2010). Based on the co-transfection experiments in primary neurons (Figures 3G-K) and because of differences in expression patterns between $\Delta$ tau in $\Delta$ tau74 mice and FynCA in FynCA55 mice 


\section{A}

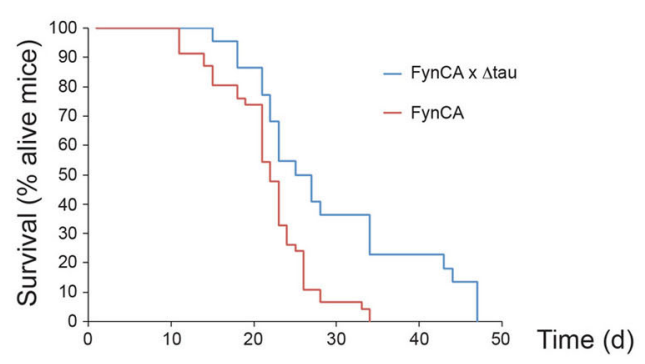

B

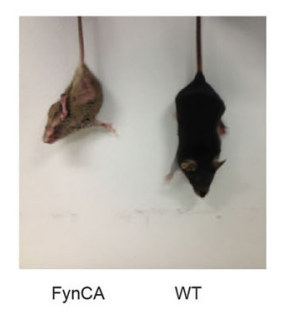

C

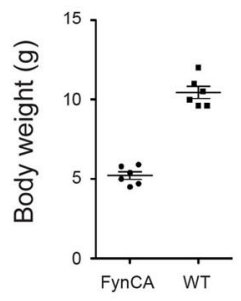

D
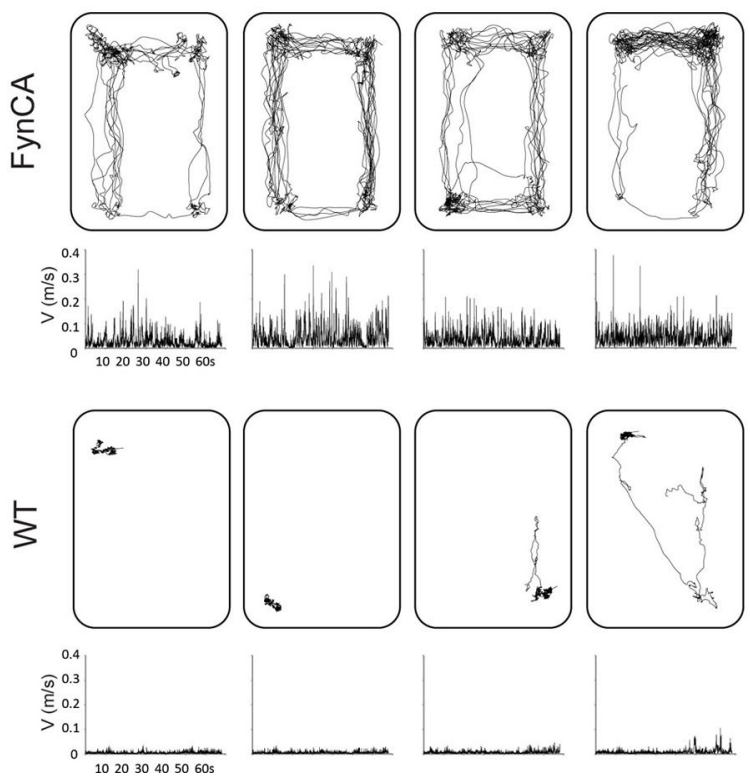

FIGURE 4 | FynCA mice are characterized by early mortality, reduced weight, and hyperactivity. Transgene-positive offspring derived from FynCA founders \#14, 35, and 55 is characterized by early lethality (A). Crossing FynCA mice with $\triangle$ tau mice results in a slight extension of the lifespan of the FynCA mice. The transgene-positive offspring derived from these lines tends to be smaller as shown for \#55 offspring (B), and to show less weight gain than the non-transgenic littermates such that by 2-3 weeks of age they weigh only half of their non-transgenic littermates (Student's $t$-test, $P<0.0001$ ) (C). Tracking of four FynCA transgenic mice (top) and four non-transgenic littermates (bottom) at 18 days of age. The FynCA mice run in circles and keep this hyperactivity up for hours unless being put back to their litter (D).

(which is in part due to different integration sites of the transgenes) (Figure 5), we expected only a subtle effect on survival. This is in fact what we observed because the rescue conferred by $\Delta$ tau on the mortality of the FynCA mice was very subtle (Figure 4A).

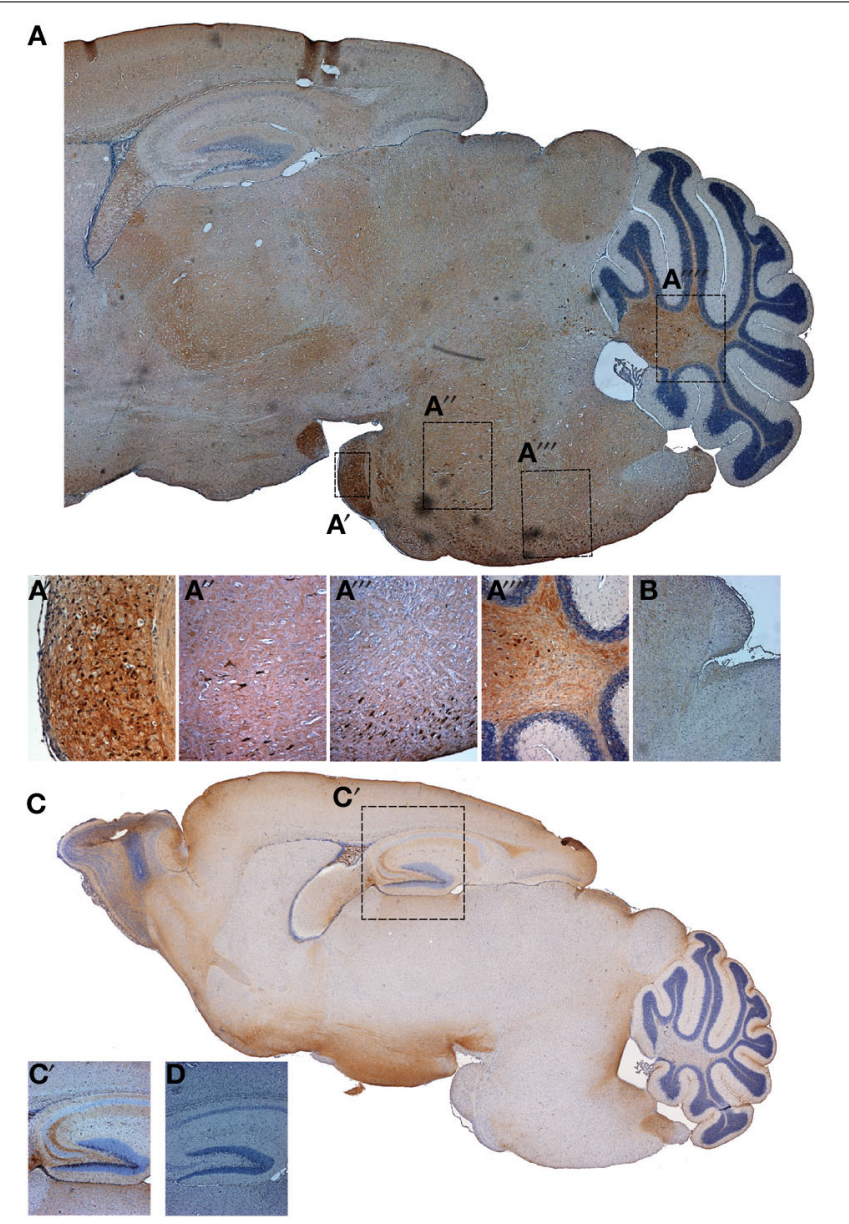

FIGURE 5 | Fyn expression in FynCA mice and tau phosphorylation. A myc-specific antibody was used to reveal Fyn expression in

FynCA-transgenic mice (A), using non-transgenic littermates as controls (B). Phosphorylation of endogenous tau was revealed using the Ser202/Thr205-specific antibody AT8, analyzing FynCA-transgenic mice (C) and non-transgenic littermate controls (D).

\section{INCREASED PHOSPHORYLATION OF NR2b AND tau IN FynCA MICE}

We next obtained synaptosomal fractions adopting a previously established protocol (Ittner et al., 2010). Different from what we had done previously (Ittner et al., 2010), we used whole forebrains rather than pooled hippocampi. We sequentially extracted synaptosomes ( $n=4$ per genotype) from wild-type, FynCA and FynCA $\times \Delta$ tau $\left(\mathrm{Tau}^{+/-}\right)$mice and analyzed the PSD fraction containing the PSD for levels of Fyn, PSD-95, the NMDA receptor subunit 2b (NR2b), NR2b phosphorylated at Y1472, tau, and tau phosphorylated at the AT8 epitope S202/T205 and at the 12E8 epitope S262(/S356), using actin and PSD95 for normalization (Figures 6A,B). We also obtained total brain extracts, and as mentioned above, found that levels of transgenic FynCA expression were approximately six-fold higher than endogenous murine Fyn (Figure 6A).

When we analyzed the PSD fraction we detected massively increased levels of Fyn in FynCA compared with wild-type mice. When FynCA mice co-expressed $\Delta$ tau, Fyn levels in the PSD 


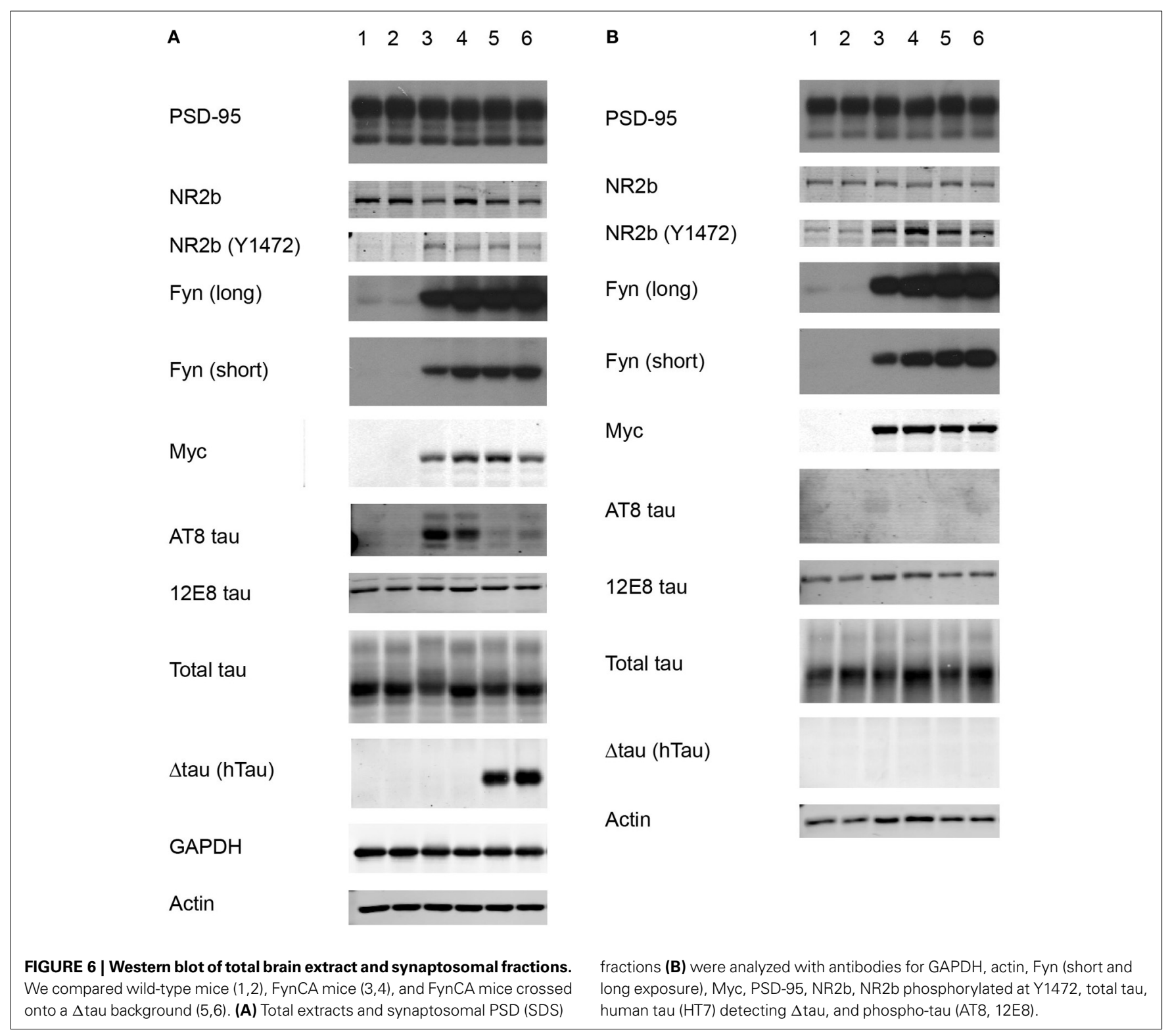

fraction were not reduced, suggesting that either high levels of Fyn override the inhibition by $\Delta$ tau in targeting Fyn to the spine (as observed for normal Fyn levels in $\Delta$ tau74 mice) or nonoverlapping expression patterns, or both. We found high levels of tau in the PSD fraction. Interestingly, whereas in total extracts obtained from FynCA mice, tau was massively phosphorylated at the AT8 epitope (tau phosphorylated at S202/ S205) (reflecting the histological data), we failed to detect tau phosphorylated at AT8 in the PSD fraction. This would suggest that if tau in the FynCA mice were phosphorylated at the AT8 epitope, levels in the spines would be very low. Interestingly, when FynCA mice were crossed onto a $\Delta$ tau background, in total brain extracts, tau phosphorylation at the AT8 epitope was strongly reduced. Note: $\Delta$ tau itself is not phosphorylated at the AT8 epitope, which is in line with previous observations showing that $\Delta$ tau is not highly phosphorylated (Klein et al., 2002; Ittner et al., 2010). In agreement with these previous data, $\Delta$ tau is not targeted to the PSD (Figure 6B). We also assessed tau phosphorylation at the 12E8 epitope (tau phosphorylated at S262 and possibly S356) and found that for the FynCA mice, 12E8 phosphorylation in the PSD fraction was increased compared with the fraction obtained from non-transgenic littermate controls. This increase was slight and not found in mice co-expressing FynCA and $\Delta$ tau.

We further assessed NR2b phosphorylation and found that Y1472 phosphorylation was approximately four-fold increased in total extracts and in the PSD fraction obtained from FynCA compared with wild-type mice, and that levels were also increased when $\Delta$ tau was co-expressed with FynCA.

\section{DISCUSSION}

Using two cellular systems we firstly showed that the palmitoylation signal is critical for the subcellular localization of the kinase 
Fyn: Mutating the two critical amino-terminal cysteine residues into serines abrogates localization of Fyn to the plasma membrane in HEK293 cells, and to dendritic spines in primary neuronal cultures. By mutating Y531 of Fyn to phenylalanine, we generated mice that express a constitutively active form of Fyn in neurons. These mice are characterized by early lethality around the age of weaning, a massively reduced weight and hyperactivity. We are also presenting preliminary evidence that constitutively active Fyn causes phosphorylation of endogenous murine tau at serine and threonine residues, as shown for the AT8 phospho-epitope of tau by both immunohistochemistry and Western blot analysis. Interestingly, tau in the spine seemed either not to be phosphorylated at AT8 at all or at least levels must be very low as we failed to detect AT8 phosphorylation in the "PSD blot" that was processed together with the "total blot."

Fyn is a kinase that has been proposed to act as a signal integration hub that is involved in a vast number of cellular programs (Wang et al., 2013). In a pathological context such as AD, Fyn has been shown to integrate signals that ultimately lead to neuronal demise. Fyn mediates the downstream toxicity of $A \beta$ by overactivating cellular receptors such as the NMDA receptor either directly or indirectly (Ittner et al., 2010). An additional role has been presented for the prion protein, $\mathrm{PrP}^{\mathrm{c}}$. It has been shown that $\mathrm{A} \beta$-induced dendritic spine loss requires both $\mathrm{PrP}^{\mathrm{c}}$ and Fyn, and that in mice lacking $\operatorname{PrP}^{\mathrm{c}}$, human familial AD transgene-induced convulsive seizures do not occur (Um et al., 2012). More recently, the metabotropic glutamate receptor 5 (mGluR5) was shown to function as a co-receptor of $\operatorname{PrP}^{\mathrm{c}}$ (Um et al., 2013). Soluble A $\beta$ was found to bind to $\mathrm{PrP}^{\mathrm{c}}$ at dendritic spines where $\operatorname{PrP}^{\mathrm{c}}$ forms a complex with Fyn, resulting in the activation of Fyn and subsequent Y18 phosphorylation, as shown with antibody anti-pY18 (Larson et al., 2012). However, how tau and Fyn are targeted to the dendritic spine is not understood. Two scenarios have been discussed previously (Larson et al., 2012): (i) Either Fyn is not bound to tau before entering the spine and activated Fyn then causes the phosphorylation of tau at Y18 resulting in its accumulation at the PSD. It is possible that at this stage phosphorylated Fyn might be bound to phosphorylated Tau binding to the SH3 domain of PSD-95, where it is ideally located to modulate NMDA receptor subunits such as NR2b at residue Y1472 (Ittner et al., 2010). (We found that FynCA is localized to the spine where it phosphorylates NR2b at Y1472.) (ii) The alternative scenario is that in the dendrite, Fyn is already bound to tau, which then translocates to the PSD to interact with PSD-95. There, another tyrosine kinase, Pyk2 (Köhler et al., 2013), could potentially activate Fyn and cause phosphorylation of tau at Y18. We found that increased levels of activated Fyn causes increased levels of phosphorylated Tau in the FynCA mice as shown for the AT8 epitope, although no increased phosphorylation was found for the spines. A time-resolved model for how $\mathrm{A} \beta$ via Fyn ultimately causes neuronal demise has been presented by Jannic Boehm, integrating the effects of $A \beta$ on Fyn and STEP, a Fyn-phosphatase that eventually inactivates Fyn (Boehm, 2013).

Co-expressing $\Delta$ tau and FynCA did not result in reduced levels of Fyn in the synaptosomal compartment. We had previously shown that transgenic expression of $\Delta$ tau, which accumulates in the soma and is prevented from entering the dendrites, traps (endogenous) Fyn in the cell body and prevents it from entering the spines (Ittner et al., 2010). When FynCA is massively overexpressed, levels of $\Delta$ tau seem not to be sufficiently high to prevent Fyn from entering the spines suggesting that manipulation of tau levels for therapeutic intervention may not be beneficial when Fyn is highly activated and/or its levels are increased, as may be the case under disease conditions. On the other hand it has been reported for $\mathrm{AD}$ brain by quantitative immunoblotting that Fyn levels were increased in the insoluble fraction and decreased in the soluble fraction. Soluble Fyn levels were directly correlated with cognitive scores and inversely correlated with taucontaining neurofibrillary tangle counts in the frontal cortex (Ho et al., 2005).

In spines it has been shown that hyperphosphorylated tau abnormally accumulates (Hoover et al., 2010). We have shown previously that in the presence of elevated levels of (phosphorylated) human tau, $A \beta$ toxicity is exaggerated, most likely because, under these conditions, more Fyn enters the spine (Ittner et al., 2010; Ittner and Götz, 2011). In the presence of oligomeric A $\beta$ tau not only changes its phosphorylation status but also alters its subcellular localization (Zempel et al., 2010, 2013). A role for tau phosphorylation specifically at the 12E8 epitope Ser262/Ser356 has been shown to be required for $A \beta$-mediated spine loss mediated via the CaMK2-AMPK signaling pathway (Mairet-Coello et al., 2013). We found in the present study that increased Fyn activity and levels in the spine do not necessarily result in increased phosphorylation, at least as shown for the AT8 epitope. This is different for the 12E8 phospho-epitope of tau, which reveals a slight increase in the FynCA PSD fraction compared to both non-transgenic littermate controls and mice co-expressing FynCA and $\Delta$ tau. Further work needs to go into how Fyn regulates tau phosphorylation and what the requirements are for dendritic and spine localization of distinct phospho-pools of tau. Whether the results of our study would have been different if we had analyzed hippocampal fractions remains to be determined.

Earlier studies have been looking into transgenic mice that expressed FynB Y531F controlled by a CaMKII/SV40 expression vector (Kojima et al., 1998). The mice presented with higher seizure susceptibility. They also showed enhanced tyrosine phosphorylation of the NR2b subunit of the NMDA receptor. This was demonstrated by immuno-precipitating the NR2b subunit from the PSD fraction, running a blot, and probing it with a phospho-tyrosine-specific antibody. We confirmed this finding by probing the PSD fraction directly with an NR2b phospho-Y1472specific antibody. Further work by Kojima and colleagues showed that an impairment of long-term potentiation in the absence of Fyn could be rescued by reintroducing FynB under control of the CamKII promoter (Kojima et al., 1997). When the constitutively active form of Fyn, Y528F was expressed in the epidermis using the keratin 14 promoter for expression (Li et al., 2007), 10\% of homozygous mice died before weaning. Otherwise the mice were normal in size, and as they aged they developed spontaneous skin tumors. Together with our findings this reveals an important role for Fyn in peripheral organs and the brain.

Further work is required to determine how Fyn and tau are transported into the spine, which posttranslational modifications govern this process and which kinases are activated by the 
tyrosine kinase Fyn in order to cause serine/threonine-directed phosphorylation of substrates such as tau.

\section{ACKNOWLEDGMENTS}

We thank Tishila Palliyaguru for immunohistochemistry; Jesus Bertran-Gonzalez for image composition; Jane Ellis for help with histology; Linda Cumner for generating the transgenic lines and her, Tishila Palliyaguru as well as Trish Hitchcock and the animal care team for animal maintenance and colony management. This study was supported by the Estate of Dr. Clem Jones AO, as well as grants from the Australian Research Council and the National Health and Medical Research Council of Australia to Jürgen Götz.

\section{SUPPLEMENTARY MATERIAL}

The Supplementary Material for this article can be found online at: http://www.frontiersin.org/journal/10.3389/fnmol. 2014.00040/abstract

\section{Supplementary Movie | Movie $(1 \mathrm{~min})$ showing two FynCA transgenic mice (bottom) and two non-transgenic littermates (top) at $\mathbf{1 8}$ days of age. The FynCA mice run in circles and keep up this hyperactivity for hours unless being put back to their litter.}

\section{REFERENCES}

Bhaskar, K., Hobbs, G. A., Yen, S. H., and Lee, G. (2010). Tyrosine phosphorylation of tau accompanies disease progression in transgenic mouse models of tauopathy. Neuropathol. Appl. Neurobiol. 36, 462-477. doi: 10.1111/j.13652990.2010.01103.x

Bloom, G. S. (2014). Amyloid- $\beta$ and Tau: the trigger and bullet in Alzheimer disease pathogenesis. JAMA Neurol. 71, 505-508. doi: 10.1001/jamaneurol. 2013.5847

Boehm, J. (2013). A 'danse macabre': tau and Fyn in STEP with amyloid beta to facilitate induction of synaptic depression and excitotoxicity. Eur. J. Neurosci. 37, 1925-1930. doi: 10.1111/ejn.12251

Braak, E., Braak, H., and Mandelkow, E. M. (1994). A sequence of cytoskeleton changes related to the formation of neurofibrillary tangles and neuropil threads. Acta Neuropathol. 87, 554-567. doi: 10.1007/BF00293315

Chen, F., David, D., Ferrari, A., and Götz, J. (2004). Posttranslational modifications of tau - Role in human tauopathies and modeling in transgenic animals. Curr. Drug Targets 5, 503-515. doi: 10.2174/1389450043345236

Chin, J., Palop, J. J., Puolivali, J., Massaro, C., Bien-Ly, N., Gerstein, H., et al. (2005). Fyn kinase induces synaptic and cognitive impairments in a transgenic mouse model of Alzheimer's disease. J. Neurosci. 25, 9694-9703. doi: 10.1523/JNEUROSCI.2980-05.2005

Chin, J., Palop, J. J., Yu, G. Q., Kojima, N., Masliah, E., and Mucke, L. (2004). Fyn kinase modulates synaptotoxicity, but not aberrant sprouting, in human amyloid precursor protein transgenic mice. J. Neurosci. 24, 4692-4697. doi: 10.1523/JNEUROSCI.0277-04.2004

Deters, N., Ittner, L. M., and Götz, J. (2008). Divergent phosphorylation pattern of tau in P301L tau transgenic mice. Eur. J. Neurosci. 28, 137-147. doi: 10.1111/j.1460-9568.2008.06318.x

Götz, J., Xia, D., Leinenga, G., Chew, Y. L., and Nicholas, H. (2013). What renders TAU toxic. Front. Neurol. 4:72. doi: 10.3389/fneur.2013.00072

Haass, C., and Mandelkow, E. (2010). Fyn-tau-amyloid: a toxic triad. Cell 142, 356-358. doi: 10.1016/j.cell.2010.07.032

Ho, G. J., Hashimoto, M., Adame, A., Izu, M., Alford, M. F., Thal, L. J., et al. (2005). Altered p59Fyn kinase expression accompanies disease progression in Alzheimer's disease: implications for its functional role. Neurobiol. Aging 26, 625-635. doi: 10.1016/j.neurobiolaging.2004.06.016

Hoover, B. R., Reed, M. N., Su, J., Penrod, R. D., Kotilinek, L. A., Grant, M. K., et al. (2010). Tau mislocalization to dendritic spines mediates synaptic dysfunction independently of neurodegeneration. Neuron 68, 1067-1081. doi: 10.1016/j.neuron.2010.11.030

Ittner, L. M., and Götz, J. (2007). Pronuclear injection for the generation of transgenic mice. Nat. Protoc. 2, 1206-1215. doi: 10.1038/nprot.2007.145
Ittner, L. M., and Götz, J. (2011). Amyloid-beta and tau - a toxic pas de deux in Alzheimer's disease. Nat. Rev. Neurosci. 12, 65-72. doi: 10.1038/nrn2967

Ittner, L. M., Ke, Y. D., Delerue, F., Bi, M., Gladbach, A., van Eersel, J., et al. (2010). Dendritic function of tau mediates amyloid-beta toxicity in Alzheimer's disease mouse models. Cell 142, 387-397. doi: 10.1016/j.cell.2010.06.036

Kins, S., Crameri, A., Evans, D. R., Hemmings, B. A., Nitsch, R. M., and Götz, J. (2001). Reduced PP2A activity induces hyperphosphorylation and altered compartmentalization of tau in transgenic mice. J. Biol. Chem. 276, 38193-38200. doi: 10.1074/jbc.M102621200

Klein, C., Kramer, E. M., Cardine, A. M., Schraven, B., Brandt, R., and Trotter, J. (2002). Process outgrowth of oligodendrocytes is promoted by interaction of fyn kinase with the cytoskeletal protein tau. J. Neurosci. 22, 698-707.

Köhler, C., Dinekov, M., and Götz, J. (2013). Active glycogen synthase kinase-3 and tau pathology-related tyrosine phosphorylation in pR5 human tau transgenic mice. Neurobiol. Aging 34, 1369-1379. doi: 10.1016/j.neurobiolaging.2012.11.010

Kojima, N., Ishibashi, H., Obata, K., and Kandel, E. R. (1998). Higher seizure susceptibility and enhanced tyrosine phosphorylation of N-methyl-D-aspartate receptor subunit 2B in fyn transgenic mice. Learn. Mem. 5, 429-445.

Kojima, N., Wang, J., Mansuy, I. M., Grant, S. G., Mayford, M., and Kandel, E. R. (1997). Rescuing impairment of long-term potentiation in fyn-deficient mice by introducing Fyn transgene. Proc. Natl. Acad. Sci. U.S.A. 94, 4761-4765. doi: 10.1073/pnas.94.9.4761

Kramer-Albers, E. M., and White, R. (2011). From axon-glial signalling to myelination: the integrating role of oligodendroglial Fyn kinase. Cell. Mol. Life Sci. 68, 2003-2012. doi: 10.1007/s00018-010-0616-z

Larson, M., Sherman, M. A., Amar, F., Nuvolone, M., Schneider, J. A., Bennett, D. A., et al. (2012). The complex PrP(c)-Fyn couples human oligomeric Abeta with pathological tau changes in Alzheimer's disease. J. Neurosci. 32, 16857-16871a. doi: 10.1523/JNEUROSCI.1858-12.2012

Lashuel, H. A., Overk, C. R., Oueslati, A., and Masliah, E. (2013). The many faces of alpha-synuclein: from structure and toxicity to therapeutic target. Nat. Rev. Neurosci. 14, 38-48. doi: 10.1038/nrn3406

Lee, G., Newman, S. T., Gard, D. L., Band, H., and Panchamoorthy, G. (1998). Tau interacts with src-family non-receptor tyrosine kinases. J. Cell Sci. 111, 3167-3177.

Li, W., Marshall, C., Mei, L., Gelfand, J., and Seykora, J. T. (2007). Srcasm corrects Fyn-induced epidermal hyperplasia by kinase down-regulation. J. Biol. Chem. 282, 1161-1169. doi: 10.1074/jbc.M606583200

Mairet-Coello, G., Courchet, J., Pieraut, S., Courchet, V., Maximov, A., and Polleux, F. (2013). The CAMKK2-AMPK kinase pathway mediates the synaptotoxic effects of Abeta oligomers through Tau phosphorylation. Neuron 78, 94-108. doi: 10.1016/j.neuron.2013.02.003

Nada, S., Yagi, T., Takeda, H., Tokunaga, T., Nakagawa, H., Ikawa, Y., et al. (1993). Constitutive activation of Src family kinases in mouse embryos that lack Csk. Cell 73, 1125-1135. doi: 10.1016/0092-8674(93)90642-4

Ostareck-Lederer, A., Ostareck, D. H., Cans, C., Neubauer, G., Bomsztyk, K., Superti-Furga, G., et al. (2002). c-Src-mediated phosphorylation of hnRNP K drives translational activation of specifically silenced mRNAs. Mol. Cell. Biol. 22, 4535-4543. doi: 10.1128/MCB.22.13.4535-4543.2002

Rawat, A., and Nagaraj, R. (2010). Determinants of membrane association in the SH4 domain of Fyn: roles of N-terminus myristoylation and side-chain thioacylation. Biochim. Biophys. Acta 1798, 1854-1863. doi: 10.1016/j.bbamem.2010.06.009

Roskoski, R. Jr. (2004). Src protein-tyrosine kinase structure and regulation. Biochem. Biophys. Res. Commun. 324, 1155-1164. doi: 10.1016/j.bbrc.2004.09.171

Sandilands, E., Brunton, V. G., and Frame, M. C. (2007). The membrane targeting and spatial activation of Src, Yes and Fyn is influenced by palmitoylation and distinct RhoB/RhoD endosome requirements. J. Cell Sci. 120, 2555-2564. doi: $10.1242 /$ jcs. 003657

Sette, C., Paronetto, M. P., Barchi, M., Bevilacqua, A., Geremia, R., and Rossi, P. (2002). Tr-kit-induced resumption of the cell cycle in mouse eggs requires activation of a Src-like kinase. EMBO J. 21, 5386-5395. doi: 10.1093/emboj/cdf553

Sontag, J. M., Nunbhakdi-Craig, V., White, C. L. 3rd., Halpain, S., and Sontag, E. (2012). The protein phosphatase PP2A/Balpha binds to the microtubuleassociated proteins Tau and MAP2 at a motif also recognized by the kinase Fyn: implications for tauopathies. J. Biol. Chem. 287, 14984-14993. doi: 10.1074/jbc.M111.338681 
Tucker, K. L., Meyer, M., and Barde, Y. A. (2001). Neurotrophins are required for nerve growth during development. Nat. Neurosci. 4, 29-37. doi: 10.1038/82868

Um, J. W., Kaufman, A. C., Kostylev, M., Heiss, J. K., Stagi, M., Takahashi, H., et al. (2013). Metabotropic glutamate receptor 5 is a coreceptor for Alzheimer abeta oligomer bound to cellular prion protein. Neuron 79, 887-902. doi: 10.1016/j.neuron.2013.06.036

Um, J. W., Nygaard, H. B., Heiss, J. K., Kostylev, M. A., Stagi, M., Vortmeyer, A., et al. (2012). Alzheimer amyloid-beta oligomer bound to postsynaptic prion protein activates Fyn to impair neurons. Nat. Neurosci. 15, 1227-1235. doi: 10.1038/nn.3178

Usardi, A., Pooler, A. M., Seereeram, A., Reynolds, C. H., Derkinderen, P., Anderton, B., et al. (2011). Tyrosine phosphorylation of tau regulates its interactions with Fyn SH2 domains, but not SH3 domains, altering the cellular localization of tau. FEBS J. 278, 2927-2937. doi: 10.1111/j.1742-4658.2011. 08218.x

Wang, H., Ren, C. H., Gunawardana, C. G., and Schmitt-Ulms, G. (2013). Overcoming barriers and thresholds - signaling of oligomeric Abeta through the prion protein to Fyn. Mol. Neurodegener. 8, 24. doi: 10.1186/1750-1326-8-24

Wolven, A., Okamura, H., Rosenblatt, Y., and Resh, M. D. (1997). Palmitoylation of p59fyn is reversible and sufficient for plasma membrane association. Mol. Biol. Cell 8, 1159-1173. doi: 10.1091/mbc.8.6.1159

Zamora-Leon, S. P., Lee, G., Davies, P., and Shafit-Zagardo, B. (2001). Binding of Fyn to MAP-2c through an SH3 binding domain. Regulation of the interaction by ERK2. J. Biol. Chem. 276, 39950-39958. doi: 10.1074/jbc.M107807200
Zempel, H., Luedtke, J., Kumar, Y., Biernat, J., Dawson, H., Mandelkow, E., et al. (2013). Amyloid-beta oligomers induce synaptic damage via Tau-dependent microtubule severing by TTLL6 and spastin. EMBO J. 32, 2920-2937. doi: 10.1038/emboj.2013.207

Zempel, H., Thies, E., Mandelkow, E., and Mandelkow, E. M. (2010). Abeta oligomers cause localized $\mathrm{Ca}(2+)$ elevation, missorting of endogenous Tau into dendrites, Tau phosphorylation, and destruction of microtubules and spines. J. Neurosci. 30, 11938-11950. doi: 10.1523/JNEUROSCI.2357-10.2010

Conflict of Interest Statement: The authors declare that the research was conducted in the absence of any commercial or financial relationships that could be construed as a potential conflict of interest.

Received: 05 March 2014; accepted: 22 April 2014; published online: 13 May 2014. Citation: Xia D and Götz J (2014) Premature lethality, hyperactivity, and aberrant phosphorylation in transgenic mice expressing a constitutively active form of Fyn. Front. Mol. Neurosci. 7:40. doi: 10.3389/fnmol.2014.00040 This article was submitted to the journal Frontiers in Molecular Neuroscience.

Copyright (C) 2014 Xia and Götz. This is an open-access article distributed under the terms of the Creative Commons Attribution License (CC BY). The use, distribution or reproduction in other forums is permitted, provided the original author(s) or licensor are credited and that the original publication in this journal is cited, in accordance with accepted academic practice. No use, distribution or reproduction is permitted which does not comply with these terms. 